\title{
Recovery of function after brain damage: The benefits of diets supplemented with the calcium channel blocker nimodipine
}

\author{
T. E. LEVERE, K. FORD, and M. SANDIN \\ North Carolina State University, Raleigh, North Carolina
}

\begin{abstract}
The acute administration of the dihydropyridine calcium channel blocker nimodipine has been shown to have a number of benefits with regard to recovery of function following brain injury and the behavioral dysfunctions associated with aging. On the basis of these results, we tested whether the chronic administration of the drug as a dietary supplement might also facilitate the recovery of a learned behavior following neocortical brain injury. The results of an initial investigation using diets containing $300 \mathrm{ppm}$ of nimodipine suggested that this strategy was ineffective in changing the progress of recovery of function. The present report describes a similar paradigm but with an increased concentration of nimodipine in the diet. The results indicated that diets high in nimodipine $(1,000 \mathrm{ppm})$ throughout the experimental procedure, or simply after the neocortical injury, were associated with significant postoperative savings of a preoperatively learned visual discrimination.
\end{abstract}

The acute administration of the dihydropyridine calcium channel blocker nimodipine appears to be beneficial in decreasing the consequences of neocortical injury in young individuals. For example, if nimodipine is administered within $12 \mathrm{~h}$ of cerebral ischemia, it will significantly reduce cell loss by preventing calcium overload (see Scriabine, Schuurman, \& Traber, 1989, for a review). Additionally, if rats are given nimodipine prior to each day's postoperative retraining, then they will recover a preoperatively learned brightness discrimination with $40 \%$ fewer errors than operated animals not given the drug (LeVere, Brugler, Sandin, \& Gray-Silva, 1989).

The acute administration of nimodipine would also appear to mitigate certain behavioral dysfunctions that are associated with aging. In this regard, aged, but not young, rabbits will perform better in a trace-conditioning paradigm if given nimodipine than will untreated animals (Deyo, Straube, \& Disterhoft, 1989). Furthermore, trial-specific memory is improved in both aged rats (LeVere \& Walker, 1991) and aged monkeys (Sandin, Jasmin, \& LeVere, 1990) when administered nimodipine prior to testing. Thus the acute administration of this dihydropyridine would seem to be a viable possible treatment strategy for the CNS pathologies associated with both injury and aging.

In a somewhat more general behavioral investigation, Schuurman and Traber (1989; also see a review by Scriabine et al., 1989) have reported improvement in aged rats on a number of unlearned behavioral measures when

This research was supported by a gift from the Division of Preclinical Pharmacology, Miles Laboratories, to T.E.L. Correspondence and requests for reprints should be addressed to T. E. LeVere, Behavioral Neurobiology Laboratory, Department of Psychology, Box 7801, North Carolina State University, Raleigh, NC 27695-7801. the animals were treated with nimodipine. An interesting procedural aspect of this research was that the drug was administered chronically in the animals' food as opposed to the acute dosing procedures used in the investigations described above.

A question that then becomes of some importance is whether dietary supplements of nimodipine might also benefit recovery of function in young animals that have sustained neocortical injury. We initially tested this possibility by feeding young rats diets containing $300 \mathrm{ppm}$ of nimodipine both before and after their brain injuries. Unfortunately, we were unable to detect even the slightest effect of this diet on the recovery of a preoperatively learned brightness habit. It is possible, however, that these null results were due to the relatively low concentration of nimodipine in the animals' food. In this regard, Schuurman et al. used diets containing $1,000 \mathrm{ppm}$ of nimodipine, so we replicated our initial effort but with similar concentrations of nimodipine, and the results, which we report here, are more positive.

\section{METHOD}

\section{Subjects}

The subjects were 38 male hooded rats born in our laboratory colony, which is temperature controlled and maintained on a reversed 14:10-h day:night cycle. The animals were housed in groups prior to the experiment (at 90-120 days of age), when they were individually caged. During the experiment, the animals were allowed free access to food and water; however, as described in the Procedure section, the food pellets sometimes contained a supplement of nimodipine.

\section{Apparatus}

The apparatus was our computer-controlled Rat Automated Training System (RATS), which has been previously described in detail 
(see LeVere \& Fontaine, 1978). Briefly, the apparatus is an equilateral $\mathrm{Y}$-maze with walls and ceiling fabricated from plastic and a floor of brass rods, which form a shock grid. Translucent panels at the end of each arm of the Y-maze can be illuminated by a $7-\mathrm{W}, 110-\mathrm{V}$ ac bulb, or not illuminated, to produce the brightness cues the animal must learn to discriminate. The maze itself is housed in a large acoustical chamber to mask external sounds.

Operationally, the apparatus is used in a discrete trial mode. A trial begins when the brightness of the rear panels change and there is the initiation of a mild pulsating footshock ( $1 \mathrm{sec}$ on, $1 \mathrm{sec}$ off; intensity between 0.1 and $0.5 \mathrm{~mA}$, depending on the individual animal's response to footshock). All animals were trained to escape footshock by entering the alley with the lighted rear panel. Thus a trial began when the light at the rear of the alley occupied by the rat was extinguished and the rear panel of one of the two unoccupied alleys was illuminated. The rat was then required to enter that alley and interrupt a photocell circuit some 5 in. from the end of the alley. If the animal entered an incorrect alley, that is, a dark alley, then the trial was scored by the computer as incorrect but continued until the animal entered the correct, lighted alley. Once in the correct alley, a 30-sec intertrial interval (ITI) began, during which time the cue conditions remained constant. During the ITI, the animal was required to remain in the alley; any attempt to enter another alley suspended the ITI and reinstated the pulsating footshock until the animal returned to the lighted alley, at which time the ITI was resumed.

The pellets the animals were fed were manufactured by Dyets (Bethlehem, PA) from the same run lot of Purina (No. 5001) powered rat chow. Half of the pellets were supplemented with nimodipine $(1,000 \mathrm{ppm})$, and the remaining pellets did not contain nimodipine. Thus, with the exception of nimodipine, the pellets were identical.

\section{Procedure}

The experimental paradigm involved four groups. Two of these groups were maintained on a constant diet throughout the experiment, and the other two had their diets switched at the time of surgery. Group NN $(n=9)$ was fed nimodipine-enriched pellets during original learning, during postoperative recovery, and during postoperative retraining. Group PP $(n=10)$ was fed plain pellets throughout the experiment. Group PN $(n=8)$ was maintained on plain pellets during original learning but then switched to the nimodipine-supplemented pellets during postoperative recovery and postoperative retraining. The pre- and postoperative diets of Group NP $(n=11)$ were just the reverse of those of Group PN.

The animals in each group began their particular diet 2 weeks prior to any training. Following this, the animals learned the twochoice brightness discrimination according to the procedure described above. Training progressed at the rate of 10 trials per day, 5 days per week, until an animal reached a criterion of at least nine first-choice-correct responses within a day's training.

Within $\mathbf{4 8} \mathrm{h}$ of preoperative criterion performance, each animal was subjected to visual decortication. The lesion was accomplished by aspiration under deep sodium pentobarbital anesthesia and was intended to include all neocortex receiving visual projections from the thalamus. Thus, all neocortex posterior to a bilateral line beginning at bregma and becoming tangential to the rhinal sulcus was removed. After surgery, each animal was given a prophylactic injection of Dual-Pen (penicillin $G$ benzathine and penicillin $G$ procaine in aqueous suspension) and returned to its home cage for 2 weeks prior to postoperative retraining. During the first few days of this postoperative rest, the animal's diet of pellets was supplemented with nimodipine or plain wet mash, depending on the treatment condition.

Postoperative retraining was identical to preoperative original learning, except for the diet change in two of the groups, and was carried to the same behavioral criterion.

\section{Histology}

When postoperative training was completed, the rats were terminally anesthetized and perfused through the heart with normal saline followed by $10 \%$ buffer formalin. The rats' brains were removed, and the boundaries of the lesions were drawn on surface diagrams with the aid of a dissecting microscope. The percentage of neocortical injury was then determined from these diagrams by planimetry. The brains of the animals with the largest and smallest lesions in each group were then sunk in buffer formalin plus $30 \%$ sucrose and sectioned at $50 \mu$ on a freezing microtome. Every fourth section through the neocortical injury and the dorsal lateral geniculate nucleus was saved and stained with cresyl violet for microscopic evaluation.

\section{RESULTS}

\section{Histology}

The mean neocortical injury for Groups NN, PP, NP, and PN was $47.2 \%, 45.9 \%, 45.8 \%$, and $46.0 \%$, respectively. An analysis of variance (ANOVA) computed on these data indicated no significant differences between the groups $(F<1)$. In all cases, all six layers of the neocortex had been removed and the lesions were of the extent intended at the time of surgery.

Microscopic analysis of the cresyl violet material indicated complete degeneration of principal cells within the dorsal lateral geniculate nucleus in all cases. The ventral lateral geniculate nucleus appeared normal. As would be expected from lesions of this size, there was considerable loss of the corpus callosum and some cell loss in the posterior thalamic nucleus and pretectal area. However, there were no visually detectable differences between the groups. Also, comparison of the present material with other brains we have processed showed no significant differences in other brain areas within the limits of the sections used for the present analysis.

\section{Behavior}

We were unable to detect any feeding differences between the groups maintained on plain pellets or pellets supplemented with nimodipine, and all animals consumed similar amounts of food and gained similar amounts of weight. There were no general behavioral changes associated with either diet.

Table 1 provides the mean preoperative original learning, postoperative recovery scores, and percent savings for each group. ANOVAs on the learning and recovery scores were not significant. However, percent savings, which accounts for an individual's postoperative recovery with regard to its preoperative original learning, was quite different.

Percent savings, defined as

$$
\frac{\text { (Preoperative learning) }- \text { (Postoperative retraining) }}{\text { Preoperative learning }} \times 100 \text {, }
$$

demonstrated that diet did have significant consequences for recovery following brain injury (see Figure 1). All of the animals in Group NN showed positive savings, that is, each performed better postoperatively than preopera- 
Table 1

Mean Incorrect Trials and Percent Savings

\begin{tabular}{ccrcccc}
\hline & \multicolumn{2}{c}{ Trials } & & \multicolumn{2}{c}{ SEM } & \\
\cline { 2 - 3 } \cline { 5 - 6 } Group & Pre & Post & & Pre & Post & Savings \\
\hline NN & 27.2 & 14.3 & & 3.3 & 3.2 & 46.1 \\
PN & 23.0 & 8.5 & & 3.2 & 2.8 & 49.3 \\
NP & 23.4 & 13.3 & & 4.3 & 2.2 & 24.0 \\
PP & 25.6 & 17.2 & & 3.9 & 2.7 & 13.6 \\
\hline
\end{tabular}

Note - Pre $=$ preoperative training; Post $=$ postoperative training; $S E M= \pm$ standard error of the mean.

tively. In Group PN group, 87\% of the animals showed positive savings. On the other hand, in Groups NP and PP, the percentage of animals showing positive savings dropped to $72 \%$ and $50 \%$, respectively. Clearly, if an individual animal's postoperative performance is considered in light of its preoperative capacities, then what the animal eats is of considerable importance. The chi-square statistic confirmed this conclusion $\left(\chi^{2}=8.01, d f=3\right.$, $p<.05)$.

\section{DISCUSSION}

Several years ago, we used the present paradigm to investigate whether a diet supplemented with the dihydropyridine nimodipine might mitigate the consequences of brain injury. The impetus for this research was based on our finding that the acute postoperative administration of this drug significantly reduced the number of errors committed when a brain-injured rat recovered a preoperatively learned behavior. However, our attempt to replicate these results with modified diets was less than satisfying. We were unable to detect any effects when the animals were fed a diet containing a supplement of $300 \mathrm{ppm}$ of nimodipine as compared with animals maintained on a diet without nimodipine. Thus, either our original findings with acute postoperative administration of nimodipine were in error, or we miscalculated the dietary concentration of nimodipine.

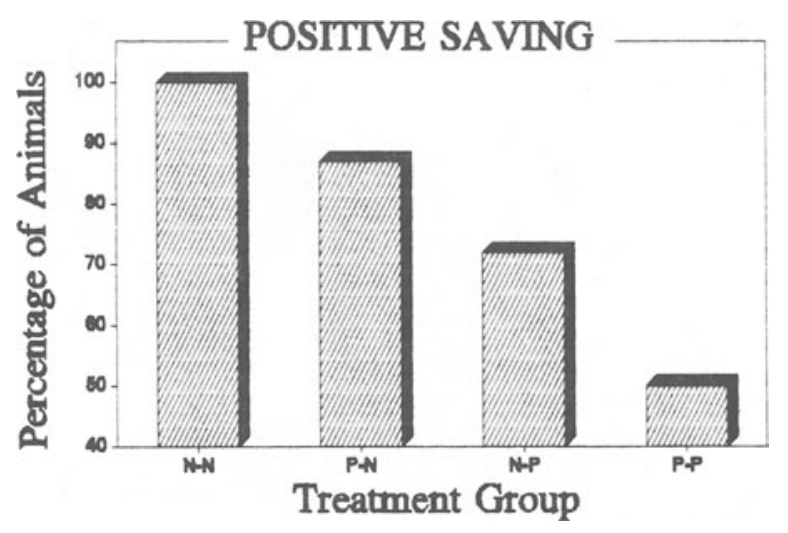

Figure 1. Percentage of animals in each group showing positive savings.
Almost concurrently, however, there was evidence that dietary supplements of nimodipine could alleviate some of the deficits associated with aging (Schuurman \& Traber, 1989). The difference between their data and our more negative result was that the concentration of nimodipine used by Schuurman and Traber was over three times higher than what we had used. On these grounds, our decision to experiment with diets containing only $300 \mathrm{ppm}$ was, perhaps, in error.

Accordingly, we replicated our previous paradigm in the present study but used diets containing a supplement of $1,000 \mathrm{ppm}$ of nimodipine. Although the results were not as dramatic as our previous experiments in which nimodipine was administered acutely during postoperative retraining (LeVere et al., 1989), they were nonetheless impressive. For example, all of the animals fed the nimodipine-supplemented diet throughout the experimental procedure (Group NN) showed positive savings during postoperative retraining. On the other hand, only $50 \%$ of the animals fed a diet of plain pellets during the experiment (Group PP) showed positive postoperative savings.

But perhaps of even more interest is the data from the groups that switched diets at the time of surgery. Of the group that was fed a plain diet preoperatively and then switched to the nimodipine-supplemented diet at the time of surgery (Group PN), 87\% showed positive postoperative savings. However, only $72 \%$ of the animals that were fed a nimodipine-supplemented diet 2 weeks prior to and during original learning but then, at the time of surgery, were switched to a plain diet (Group NP) showed positive postoperative savings. The implication of these data is that a nimodipine-enriched diet may not be a strong prophylactic with respect to the behavioral consequences of neocortical brain injury but may be consistently beneficial if initiated after brain injury. The mechanism that controls this is, at present, not clear, but at least two possibilities should be considered.

First, nimodipine, when administered within $12 \mathrm{~h}$ of cerebral ischemia, can significantly reduce cell loss by preventing calcium overload (see Scriabine et al., 1989, for a review). Notwithstanding this, we were unable to histologically verify any decrease in cell loss in our cresyl violet material. Moreover, the animals in Group NP, which were fed a nimodipine-supplemented diet prior to surgery, should have been protected if cell loss was the controlling variable. Yet, while these animals were clearly superior to Group PP, they did not show the savings of Group PN. Accordingly, we believe that a reduction of cell loss following neocortical injury is not, in this case, the critical factor.

An alternative to the notion of cell survival is that the calcium-channel-blocking properties of nimodipine may facilitate neural functioning independent of the drug's ability to mitigate cell death. For example, nimodipine appears effective in aiding recovery of function even when initially administered some 2 weeks after brain injury, when cell loss is probably no longer an issue (LeVere et al., 1989). In addition, nimodipine is effective in re- 
ducing the short-term memory deficits associated with aging, but only when the drug is systematically active (LeVere \& Walker, 1991; Sandin et al., 1990). Again, if the drug's potential to reduce cell death was the controlling factor in the latter studies of aged animals, then one would expect its effect to last beyond the drug treatment, and simply because more neural cells would be alive.

Thus, we suggest that the present results may be due to the possibility, as detailed by LeVere and Walker (1991), that nimodipine can improve the functioning of neural cells that survive neural injury by normalizing the operation of damaged but surviving neural cells and systems. We recognize that this is a speculation, but it is compatible with analysis of the consequences of brain injury on learned behaviors (LeVere, 1975; LeVere, 1980; LeVere \& LeVere, 1982) as well as nimodipine's effect on the cognitive dysfunctions associated with aging.

\section{REFERENCES}

Deyo, R. A., Straube, K. T., \& Disterhoft, J. F. (1989). Nimodipine facilitates associative learning in aging rabbits. Science, 243, 809-811.

LeVere, N. D., LeVere, T. E. (1982). Recovery of function after brain damage: Support for the compensation theory of the behavioral deficit. Physiological Psychology, 10, 165-174.

LEVERE, T. E. (1975). Neural stability, sparing, and behavioral rocovery following brain damage. Psychological Review, 82, 344-358.

LEVERE, T. E. (1980). Recovery of function after brain damage: A theory of the behavioral deficit. Physiological Psychology, 8, 297-308.

LeVere, T. E., Brugler, T., SAndin, M., aray-Silva, S. (1989). Recovery of function after brain damage: Facilitation by the calcium entry blocker nimodipine. Behavioral Neuroscience, 103, 561-565.

LeVere, T. E., \& Fontaine, C. W. (1978). A demonstration of the importance of RNA metabolism for the acquisition but not performance of learned behaviors. Experimental Neurology, 59, 444-449.

LEVERE, T. E., WALKER, A. (1991). Old age and cognition: Enhancement of recent memory in aged rats by the calcium channel blocker nimodipine. Neurobiology of Aging, 13, 63-66.

SAndin, M., Jasmin, S., L LeVere, T. E. (1990). Aging and cognition: Facilitation of recent memory in aged nonhuman primates by nimodipine. Neurobiology of Aging, 11, 573-575.

SchuUrman, T., \& Traber, J. (1989). Old rats as an animal model for senile dementia: Behavioral effects of nimodipine. In M. Bergener \& B. Reisberg (Eds.), Diagnosis and treatment of senile dementia, (pp. 295-307). Berlin: Springer-Verlag.

Scriabine, A., Schuurman, T., \& Traber, J. (1989). Pharmacological basis for the use of nimodipine in central nervous system disorders. FASEB Journal, 3, 1799-1806.

(Manuscript received October 11, 1991; revision accepted for publication February 28, 1992.) 